\title{
Establishment and early history of the astrophysics division
}

\author{
Chul Hoon Lee ${ }^{1}$
}

Received: 30 October 2020 / Accepted: 3 November 2020 / Published online: 8 April 2021

(c) The Korean Physical Society 2021

Sixty-eight years have passed since the Korean Physical Society (KPS) was founded in Busan in 1952 during the Korean War. Divisions representing specialized research fields were established successively in the Society. The first was the Particle Physics Division established in 1967, and a total of 12 divisions have been established so far. The Astrophysics Division, the eleventh of the twelve, was established in October 1995, and we celebrate its 25th anniversary this year. The KPS decided to publish a special issue to commemorate this anniversary, and the editorial board of the Journal of Korean Physical Society (JKPS) asked me to write an article titled "Establishment and Early History of the Astrophysics Division". As the chairman of the first and the second term steering committees of the Division, I felt obliged to accept the request, but I also worried a little because my memory of the exact dates of the events and the persons involved in them is not very vivid, and I do not have much reference material. In fact, I used to keep many documents produced in the process of establishing the Division, but I disposed of most of them when I had to empty my office at the time of my retirement from Hanyang University as I thought not getting too attached to old material would be wise. I never thought I would get to miss them, but now I do. Now, as I am to describe the process of establishing the Division and the early appearance of the Division, all I can do is to recollect those events and persons.

In the early 1990s, researchers in the field of Astrophysics discussed the necessity of having the Astrophysics Division in KPS. Until then, their academic activities were carried out through the Particle Physics Division and the Nuclear Physics Division of KPS, the Korean Astronomy Society, etc. In the study of phenomena associated with astronomical objects and the Universe, knowledge in various fields, such as particle physics, nuclear physics, general relativity, and statistical physics, is needed. On the other hand,

Chul Hoon Lee

chulhoon@hanyang.ac.kr

1 Department of Physics, Hanyang University, Seoul, Korea researchers in the fields of particle physics and nuclear physics recognized the practical limit in the possibilities of setting up experimental facilities for verifying their theories on extremely high energy phenomena. They, therefore, started having interest in astrophysical phenomena. Also, observational techniques in astrophysics were rapidly advancing, and many experimental projects were developed to gather information on phenomena at far away sites in the Universe. These made astrophysics a much more active research field in the United States, Europe, Japan, etc. One example showing this trend is the establishment of the Astrophysics Division in 1970 in the American Physical Society (founded in 1899). The number of researchers in astrophysics in Korea in the early 1990s was not very high, but taking into account this trend in other advanced countries, I guessed that the number would grow rapidly.

The first step in efforts to establish the Astrophysics Division was taken in 1992 (an error of about a year should be allowed as my memory is not very clear) by some young researchers, including Prof. Sungwon Kim of Ewha Womans University and Dr. Doojong Song (passed away in 2020) of the Korea Astronomy and Space Science Institute (KASI), who decided to submit a petition for the establishment of the Division to KPS. They asked others to join by putting their signatures on the petition. I remember signing myself, feeling full empathy for their effort. Upon receiving the petition, the Society gave notice that the petitioners would operate independent Astrophysics sessions at the spring and the fall meetings of KPS for 2 years (again my memory on this number of years is not clear). After that period of time, the Society would make an assessment of the operation and decide whether to allow the establishment of the Division. For the first board of directors meeting of the Society after the completion of the independent sessions for the given period of time, the matter of the Astrophysics Division was on the agenda. The decision was not to allow the establishment of the Division in that year, but to reconsider the matter in the next year. I think the main reason for this negative decision was that nobody on the board could speak on the behalf of the Astrophysics Division. Also, some members seemed 
to have had worries that the presence of the Astrophysics Division would cause conflicts between the Physical and the Astronomical Societies in Korea. However, the matter easily passed at the next year's board meeting. Prof. Youngduk Kim of Sogang University, vice president of the Society at that time, attended the meeting and could speak for the Astrophysics Division. I guess his presence at the meeting was a decisive factor for the positive result. Soon after, a meeting of interested researchers (the most senior attendees were Prof. Youngduk Kim of Sogang University and Prof. Jewan Kim of Seoul National University) was held to discuss the actual organization of the Division. At the meeting, I was charged with the task of organizing the steering committee of the Division. The plan I made was as follows: Several (I do not clearly remember the exact number) committee members would be elected by postal vote. The right to vote would be given to those who pledged to become members of the Division as soon as its establishment had been completed. The chairman would be elected at the steering committee meeting, and he/she would appoint two more members to have even representation for various research areas. The term of the committee would be 2 years. The actual process followed the plan well, and dozens of researchers (not exceeding a hundred I remember) participated in the voting. The committee members thus elected had the first meeting and elected me as chairman. The establishment of the Astrophysics Division was officially finalized when the list of the steering committee members was given to the KPS.

In the beginning, the number of members of the Division was inevitably quite small. Consequently, the numbers of papers our Division presented at the spring and the fall KPS meetings were quite small, noticeably smaller than the numbers presented by the other Divisions. This may have been due to some members who, out of habit, having carried out their academic activities through other Divisions such as the Particle Physics and the Nuclear Physics Divisions. In any event, we felt the need to increase the number of papers presented at the Society meetings and decided to do something active instead of waiting passively. That is the reason we introduced focus sessions and tutorial sessions. We chose special topics currently attracting high interest and invited researchers who could present overviews of the topics in the focus sessions. Those who could give lecture-like presentations to explain the basic contents of some topics were invited to tutorial sessions. These arrangements made presentation halls better places for learning more about basic materials and for obtaining information on current hot topics. We expected that this would attract more members, particularly young researchers including students, to attend the meetings.

The Korean-Italian Joint Symposium on Relativistic Astrophysics is a biennial event at which Korean and Italian researchers in astrophysics interact with one another.
Its first meeting was held in Korea in 1987. During the next 33 years, it has been kept alive, with each side alternately taking charge of organizing a meeting in its country. Since the Astrophysics Division was established, the Korean side events of theSymposium have been held under the supervision of the Astrophysics Division.

After the completion of my 2-year term as chairman of the first steering committee, the second committee was organized using the same process as was used for the first one. At the first meeting of the second committee, the members pressed me to serve one more term as chairman. I unintentionally became chairman again and kept working as chairman.

Among the later developments in the Division, I think one of the important ones is that a balance has been reached between the theoretical and experimental parts. In the beginning, the members of the Division were all on the theoretical side. Obviously, a good environment for the advancement of physics study is one in which the theoretical and the experimental sides advance together side by side influencing each other. In the old days when Korea had a poor economy, inevitably most Korean physicists in research areas where experiments required big money would be on the theoretical side. In the late 1990s, discussions took place among some researchers in particle physics and astrophysics: "As the Korean economy has grown to a quite big size and the number of physicists has also become much larger, now is a good time to develop an experimental project of reasonable size which the Korean economy can support. It cannot be as big as the ones in the advanced countries, but if we choose carefully, it can have an impact in the international physics community". One such project eventually emerged. It was named the "Hanul" project, and its aim was to install a neutrino detector on the ground for the detection of cosmic neutrinos and to use the information thus obtained for verification of various particle and astrophysics theories. Everyone hoped that a successful performance of such a project would be a big occasion for the advancement of physics in Korea. However, unfortunately, the "Hanul" project was terminated without producing any result due to conflicts among participants in the project. Later, many Korean astrophysicists participated in big international experiment projects, and several experimental projects were started in Korea. Experimental astrophysics has become an active area of research now.

One good recent development in the Division was the establishment of the Astrophysics Award. Big efforts and contributions by many members of the Division made it possible. The first awardee was announced in 2019. As the aim of the award is to acknowledge good works by young researchers, it is and will remain a source of inspiration for young researchers. 
My wish is for the Astrophysics Division to continue to grow and ultimately become an institute leading the advancement of astrophysics not only in Korea but also in the world.
Publisher's Note Springer Nature remains neutral with regard to jurisdictional claims in published maps and institutional affiliations. 